\title{
Effects of Carnosine Supplementation on Carnosine Concentrations in Muscles and Blood Biochemical Indices of Rats
}

\author{
Hae-Chang Yi ${ }^{1}$, Mi Young Kim ${ }^{1}$, Changsun Choi ${ }^{1,2}$, Young-Nam Kim ${ }^{3}$, Chan-Kyu Han ${ }^{4}$, and Bog-Hieu Lee ${ }^{1,2 *}$ \\ ${ }^{I}$ Department of Food and Nutrition, Chung-Ang University, Anseong 456-756, Korea \\ ${ }^{2}$ School of Food Science and Technology, Chung-Ang University, Anseong 456-756, Korea \\ ${ }^{3}$ Department of Food and Nutrition, Duksung Women's University, Seoul 132-714, Korea \\ ${ }^{4}$ Biogeron Technology Research Group, Korea Food Research Institute, Seongnam 463-746, Korea
}

\begin{abstract}
This study evaluated the effects of carnosine supplementation on carnosine concentration in muscles and blood biochemical indices of rats. Thirty-two eight-week-old Sprague-Dawley male rats were randomly divided into a control group $(\mathrm{CON})$ as well as three carnosine-treated groups. The carnosine-treated groups included groups fed diets composed of $0.01 \%$ carnosine (LC), $0.1 \%$ carnosine (MC), and $1.0 \%$ carnosine (HC). Body weight gain, food intake, feed efficacy rate, protein efficacy rate, and organ weights were not significantly different among the groups. In all groups, the mean carnosine levels in gastrocnemius muscles were higher than the mean carnosine levels in soleus muscles. Carnosine concentrations in soleus muscles and gastrocnemius muscles were significantly higher in the HC group compared to all other groups $(p<0.05)$. Serum triglyceride and LDL-cholesterol concentrations in all of the carnosine-supplemented groups were significantly lower than those of the control group $(p<0.05)$, while HDL-cholesterol levels were significantly higher than those of the control group $(p<0.05)$. Aspartate aminotransferase levels in rats supplemented with carnosine were significantly higher than those of the control group. In conclusion, diets supplemented with high levels of carnosine can increase carnosine concentrations in skeletal muscles, which might contribute to increased exercise capacity. Furthermore, these findings suggest that high levels of dietary carnosine improve the lipid profile of rats by lowering blood LDL-cholesterol and increasing HDL-cholesterol levels.
\end{abstract}

Key words: carnosine, muscle carnosine, lipid profile, rats

\section{Introduction}

Carnosine ( $\beta$-alanyl-L-histidine) is a dipeptide of $\beta$-alanine and L-histidine that is found in the skeletal muscle, brain, and liver of many vertebrates (Jackson and Lenny, 1996; Plowman and Close, 1988). It has several functions including protection of membranes, $\mathrm{pH}$ buffering, metal chelating, and inhibition of oxidative protein modification by carbonyl compounds (Guiotto et al., 2005).

Exercise leads to an accumulation of metabolites with accompanying increases in free radical production ( $\mathrm{Ji}$, 1995; Mastaloudis et al., 2001). Reactive oxygen species are known to be a primary cause of exercise-induced disturbances in muscle homeostasis associated with muscle fatigue (Bailey et al., 2001; Petersen et al., 2001). Car-

\footnotetext{
*Corresponding author: Bog-Hieu Lee, Department of Food and Nutrition, Chung-Ang University, Anseong 456-756, Korea. Tel: 82-31-670-3276, Fax: 82-31-676-8741, E-mail: lbheelb@cau.ac.kr
}

nosine may act as an antioxidant in vivo and in vitro by quenching singlet oxygen and hydroxyl radicals released during exercise (Decker, 1995; Klebanov et al., 1997; Mastaloudis et al., 2001) and enhancing the antioxidative effect of vitamin E (Boldyrev, 1990). The soleus muscle is preferentially activated in the concentric phase, whereas the gastrocnemius muscle is preferentially activated in the eccentric phase and is activated at higher lengthening velocities (Nardone et al., 1989). Therefore, the high concentration of carnosine in the soleus and gastrocnemius muscles might reduce reactive oxygen and the degree of exhaustion, and increase power output during high-intensity exercise.

Some studies have shown that carnosine concentrations in rat tissues can be increased through dietary supplementation (Maynard et al., 2001) and carnosine concentrations in skeletal muscles are reduced by dietary L-histidine deficiencies (Dunnett and Harris, 1999; Tamaki et al., 1984). However, the results of dietary carnosine supple- 
mentation on carnosine concentrations in muscles are inconsistent. Tamaki et al. (1984) reported that a $0.875 \%$ carnosine dietary supplementation in rats did not increase carnosine concentrations in the heart, liver, and skeletal muscle. But, a high level (1.8\%) of carnosine supplementation resulted in significant increases of carnosine in the soleus muscle (Maynard et al., 2001).

In addition, carnosine prevents low density lipoprotein (LDL) oxidation from copper-induced oxidative damage in vitro (Bogardus and Boissonneault, 2000; Decker et al., 2001) and glucose-induced oxidative and glycative damage in animal tissues (Lee et al., 2005). However, it is not reported that the supplementary intake of carnosine change blood and lipid profile including triglycerides, total cholesterol, LDL cholesterol (LDL-C) and high density lipoprotein cholesterol (HDL-C) in vivo. Functionality of carnosine obtained from muscle foods should be evaluated in vivo model.

Therefore, the purpose of this investigation was to examine whether gradually increased carnosine supplementations $(0.01 \%, 0.1 \%$, and $1 \%)$ increase carnosine concentrations in skeletal muscles and improve lipid profiles in rats.

\section{Materials and Methods}

\section{Experimental design and animal care}

Thirty-two male Sprague-Dawley rats supplied by Hanlim Experimental Animal Laboratory (Korea) with an average initial body weight of $152.5 \pm 4.8 \mathrm{~g}$ were randomly assigned to four groups, each composed of eight rats. The rats were kept individually in stainless-steel cages in a room controlled for temperature $\left(22 \pm 1^{\circ} \mathrm{C}\right)$, relative humidity (50-60\%), and light (12-hour light/dark cycle). All experimental protocols followed established guidelines for the care and handling of laboratory animals and were approved by the Institutional Animal Ethics Committee of the Chung-Ang University. After a two week adaptation, the four groups were fed four different diets for four weeks. The control group (CON) was fed a CRF-1 diet (regular laboratory pellet chow; Oriental Yeast, Japan). The three carnosine-supplemented groups were fed three different diets containing $0.01 \%$ carnosine (low carnosine, or LC), $0.1 \%$ carnosine (medium carnosine, or MC), and $1 \%$ carnosine (high carnosine, or HC). Carnosine was purchased from Sigma (USA).

The rats were allowed free access to the experimental food and deionized water. There were no significant differences among the groups in body weight at the start of the experiment. Body weight and food consumption were measured every week, and food efficacy rate (FER) and protein efficacy rate (PER) were also calculated weekly.

After four weeks of ad libitum feeding, the animals were fasted overnight, anesthetized with ethyl ether, and sacrificed. Liver, heart, kidneys, spleen, soleus muscle, and gastrocnemius muscle were removed and weighed. Blood was collected immediately from the abdominal aorta, and serum was obtained by blood centrifugation (3,000 rpm, $\left.10 \mathrm{~min}, 4^{\circ} \mathrm{C}\right)$. All of the collected samples were stored at $-70{ }^{\circ} \mathrm{C}$ until analysis.

\section{Carnosine concentrations in muscle tissue}

All reagents used for carnosine analysis were HPLC grade. Acetonitrile and methanol were obtained from JT Baker (USA). Acetic acid and tetrahydrofuran (THF) were obtained from Merck (Germany). Carnosine was purchased from Sigma (USA).

Carnosine concentrations in muscle tissue were analyzed by a modification of Aristoy's method (Aristoy et al., 2004). The tissue sample was homogenized at 10,000 rpm with five-fold volume of triple-distilled water. The homogenate was centrifuged at $6,000 \mathrm{rpm}$ for 30 minutes at $4^{\circ} \mathrm{C}$ and the supernatant was filtered through a glasswool. The $300 \mu \mathrm{L}$ of supernatant was deproteinised by adding $900 \mu \mathrm{L}$ of methanol and left at $4^{\circ} \mathrm{C}$ for $15 \mathrm{~min}$ utes. The sample was centrifuged $\left(12,000 \mathrm{rpm}, 4^{\circ} \mathrm{C}\right)$ for three minutes and the supernatant $(20 \mu \mathrm{L})$ was analyzed for carnosine.

The HPLC system (Gilson, France) consisted of a Gilson 305 pump with fluorescence detector (Gilson 121 fluorometer). The column was a Haisil HL C18 (250×4.6 mm, Higgins Analytical, Inc., USA) and a binary mobile phase system was used. Solvent A was $515 \mathrm{~mL}$ of $50 \mathrm{mM}$ acetate buffer and $350 \mathrm{~mL}$ water. Four $\mathrm{M} \mathrm{NaOH}$ was then added until the $\mathrm{pH}$ reached 4.3, at which point $100 \mathrm{~mL}$ methanol and $30 \mathrm{~mL}$ THF were added. Solvent B was 290 $\mathrm{mL}$ of $50 \mathrm{mM}$ acetate buffer, $195 \mathrm{~mL}$ water, $495 \mathrm{~mL}$ acetonitrile, and $25 \mathrm{~mL}$ THF. The gradient was $16 \% \mathrm{~B}$, which increased to $17 \%$ in $2.5 \mathrm{~min}$, to $20 \%$ in $5 \mathrm{~min}$, to $30 \%$ in $7 \mathrm{~min}$, to $100 \%$ in $5 \mathrm{~min}$, and finally decreased to $16 \%$ in $5 \mathrm{~min}$. The flow rate was $1.3 \mathrm{~mL} / \mathrm{min}$. Excitation and emission wavelengths were 340 and $470 \mathrm{~nm}$, respectively.

\section{Biochemical analysis}

Triglyceride, total cholesterol, and HDL-C concentrations in serum were determined using enzymatic kits (Somang Co., Korea). LDL-C concentrations were calcu- 
lated by the Friedewald equation (Friedewald et al., 1972). Aspartate aminotransferase (AST) and alanine aminotransferase (ALT) were measured using an auto analyzer (SPOTCEM ${ }^{\mathrm{TM}}$, Japan).

\section{Statistical analysis}

Statistical analysis was performed with SPSS software program 12.0 (SPSS, Inc., USA). Data are presented as mean \pm SD. The effects of carnosine supplementation were determined using one-way ANOVA tests followed by post-hoc test (Duncan's multiple range test) for mean comparison. A $p$ value $<0.05$ was considered significant.

\section{Results}

\section{Body Weight, FER, and PER}

Body weight, food intake, FER, PER, and organ weights of rats are presented in Table 1. There were no significant differences in initial and final body weights among the groups $(p>0.05)$. Food intake, FER, and PER were not significantly different among the groups.

\section{Organ Weights}

Table 2 shows the organ weights of the groups. The weights of the liver, heart, kidneys, spleen, and testicles in the carnosine-supplemented groups did not significantly differ from those in the CON groups. The weight of the soleus muscle and gastrocnemius muscle were not significantly different among the groups.

\section{Concentrations of Carnosine in Muscle Tissue}

Carnosine concentrations in muscle tissues are shown in Table 3. Carnosine concentrations in the soleus muscle and gastrocnemius muscle were highest in the $\mathrm{HC}$ group $(p<0.05)$, whereas carnosine concentrations in the soleus and gastrocnemius in the LC and MC groups were not significantly different compared to the CON group.

\section{Biochemical analysis}

Biochemical indices in serum are presented in Table 4. The triglyceride concentrations of the carnosine-supplemented groups were lower than that of the CON group $(p<0.05)$, and decreased as carnosine-supplementation increased. There were no significant differences in serum total cholesterol levels among the groups. That said, the carnosine-supplemented groups had significantly higher concentrations of HDL-C and significantly lower concentrations of LDL-C compared to the CON group $(p<0.05)$.

Table 1. Body weight, food intake, feed efficacy rate (FER) and protein efficacy rate (PER) in rats fed a carnosine-supplemented diet for four weeks ${ }^{1)}$

\begin{tabular}{lrrrr}
\hline \hline Variable & CON & LC & MC & HC \\
\hline Initial body weight $(\mathrm{g})$ & $303.3 \pm 33.9$ & $302.3 \pm 20.9$ & $306.8 \pm 30.5$ & $304.2 \pm 16.2$ \\
Final body weight $(\mathrm{g})$ & $429.5 \pm 31.0$ & $421.6 \pm 21.0$ & $422.7 \pm 16.9$ & $420.9 \pm 10.0$ \\
Mean body weight (g) & $371.1 \pm 54.7$ & $366.5 \pm 51.7$ & $369.1 \pm 50.3$ & $366.3 \pm 50.6$ \\
Mean food intake (g/day) & $25.56 \pm 1.37$ & $24.89 \pm 1.16$ & $24.70 \pm 1.06$ & $27.93 \pm 1.19$ \\
FER (g gain/g feed) & $0.24 \pm 0.10$ & $0.23 \pm 0.08$ & $0.24 \pm 0.09$ & $0.23 \pm 0.09$ \\
PER (g gain/g feed) & $1.04 \pm 0.45$ & $1.03 \pm 0.33$ & $1.04 \pm 0.38$ & $1.02 \pm 0.38$ \\
\hline
\end{tabular}

${ }^{1)}$ Values are mean $\pm \mathrm{SD}$

${ }^{2)} \mathrm{CON}$, control; LC, low-dose $(0.01 \%)$ carnosine; MC, medium-dose $(0.1 \%)$ carnosine; HC, high-dose (1\%) carnosine

Table 2. Relative weights of organs in rats fed a carnosine-supplemented diet for four weeks ${ }^{1}$ )

\begin{tabular}{|c|c|c|c|c|}
\hline Variable Group $^{2)}$ & $\mathrm{CON}$ & $\mathrm{LC}$ & $\mathrm{MC}$ & $\mathrm{HC}$ \\
\hline \multicolumn{5}{|l|}{ Organs } \\
\hline Liver & $31.26 \pm 1.89$ & $32.04 \pm 2.26$ & $31.77 \pm 2.50$ & $30.28 \pm 2.48$ \\
\hline Heart & $3.34 \pm 0.34$ & $3.10 \pm 0.20$ & $3.11 \pm 0.36$ & $3.04 \pm 0.19$ \\
\hline Kidney & $3.58 \pm 0.27$ & $3.55 \pm 0.29$ & $3.54 \pm 0.29$ & $3.60 \pm 0.29$ \\
\hline Spleen & $2.02 \pm 0.35$ & $1.87 \pm 0.17$ & $1.97 \pm 0.21$ & $1.84 \pm 0.34$ \\
\hline Testicle & $3.53 \pm 0.38$ & $3.57 \pm 0.30$ & $3.77 \pm 0.34$ & $3.77 \pm 0.21$ \\
\hline \multicolumn{5}{|l|}{ Muscle } \\
\hline Soleus muscle & $0.46 \pm 0.05$ & $0.49 \pm 0.04$ & $0.47 \pm 0.04$ & $0.46 \pm 0.06$ \\
\hline Gastrocnemius muscle & $2.05 \pm 0.35$ & $1.88 \pm 0.15$ & $1.96 \pm 0.13$ & $2.00 \pm 0.17$ \\
\hline
\end{tabular}

${ }^{1)}$ Values are mean $\pm \mathrm{SD}$

${ }^{2)} \mathrm{CON}$, control; LC, low-dose $(0.01 \%)$ carnosine; MC, medium-dose $(0.1 \%)$ carnosine; HC, high-dose (1\%) carnosine 
Table 3. Carnosine concentrations in skeletal muscle of rats fed a carnosine-supplemented diet for four weeks ${ }^{1)}$

\begin{tabular}{|c|c|c|c|c|}
\hline Variable $\quad$ Group $^{2)}$ & $\mathrm{CON}$ & $\mathrm{LC}$ & $\mathrm{MC}$ & $\mathrm{HC}$ \\
\hline Soleus muscle (mg/g) & $1.52 \pm 0.23^{\mathrm{a}}$ & $1.57 \pm 0.18^{\mathrm{a}}$ & $1.56 \pm 0.36^{\mathrm{a}}$ & $2.14 \pm 0.42^{\mathrm{b}}$ \\
\hline Gastrocnemius muscle (mg/g) & $2.76 \pm 0.55^{\mathrm{a}}$ & $2.70 \pm 0.36^{\mathrm{a}}$ & $2.81 \pm 0.58^{\mathrm{a}}$ & $3.44 \pm 0.19^{b}$ \\
\hline
\end{tabular}

${ }^{1)}$ Values are mean $\pm \mathrm{SD}$

${ }^{2)} \mathrm{CON}$, control; LC, low-dose $(0.01 \%)$ carnosine; MC, medium-dose $(0.1 \%)$ carnosine; HC, high-dose (1\%) carnosine

${ }^{\mathrm{ab}}$ Different superscripts in the same row indicate that the data are significantly different per Duncan's multiple range test $(p<0.05)$.

Table 4. Biochemical indices in serum of rats fed a carnosine-supplemented diet for four weeks ${ }^{1)}$

\begin{tabular}{|c|c|c|c|c|}
\hline Variable Group $^{2)}$ & $\mathrm{CON}$ & $\mathrm{LC}$ & $\mathrm{MC}$ & $\mathrm{HC}$ \\
\hline \multicolumn{5}{|l|}{ Lipid profiles $(\mathrm{mg} / \mathrm{dL})$} \\
\hline Triglyceride & $31.50 \pm 12.4^{\mathrm{b}}$ & $28.10 \pm 16.9^{\mathrm{ab}}$ & $24.70 \pm 7.8^{\mathrm{ab}}$ & $16.00 \pm 8.2^{\mathrm{a}}$ \\
\hline Total cholesterol & $55.40 \pm 6.1$ & $56.60 \pm 10.8$ & $56.60 \pm 11.3$ & $64.80 \pm 7.1$ \\
\hline HDL-cholesterol & $22.70 \pm 11.1^{\mathrm{a}}$ & $42.20 \pm 7.4^{\mathrm{b}}$ & $46.60 \pm 10.1^{\mathrm{b}}$ & $48.70 \pm 9.9^{\mathrm{b}}$ \\
\hline LDL-cholesterol & $26.20 \pm 8.5^{\mathrm{b}}$ & $16.90 \pm 5.1^{\mathrm{ab}}$ & $17.80 \pm 7.1^{\mathrm{ab}}$ & $17.50 \pm 11.4^{\mathrm{ab}}$ \\
\hline Total protein $(\mathrm{g} / \mathrm{dL})$ & $5.88 \pm 0.60$ & $6.20 \pm 0.73$ & $6.09 \pm 0.45$ & $5.65 \pm 0.68$ \\
\hline Total bilirubin (mg/dL) & $0.40 \pm 0.11^{\mathrm{a}}$ & $0.59 \pm 0.23^{\mathrm{ab}}$ & $0.56 \pm 0.15^{\mathrm{ab}}$ & $0.63 \pm 0.22^{\mathrm{b}}$ \\
\hline $\operatorname{AST}^{3)}(\mathrm{IU} / \mathrm{L})$ & $114.50 \pm 16.80^{\mathrm{a}}$ & $175.13 \pm 30.08^{\mathrm{b}}$ & $179.43 \pm 26.49^{\mathrm{b}}$ & $218.88 \pm 40.65^{\circ}$ \\
\hline $\mathrm{ALT}^{4)}(\mathrm{IU} / \mathrm{L})$ & $11.17 \pm 2.86$ & $19.63 \pm 6.52$ & $21.00 \pm 6.73$ & $18.25 \pm 12.78$ \\
\hline Hemoglobin (g/dL) & $14.94 \pm 3.08$ & $14.76 \pm 2.75$ & $15.29 \pm 1.64$ & $14.88 \pm 1.15$ \\
\hline Hematocrit (\%) & $43.24 \pm 21.69$ & $36.79 \pm 6.61$ & $37.49 \pm 4.38$ & $38.13 \pm 2.43$ \\
\hline
\end{tabular}

${ }^{1)}$ Values are mean $\pm \mathrm{SD}$

${ }^{2)} \mathrm{CON}$, control; LC, low-dose (0.01\%) carnosine; MC, medium-dose (0.1\%) carnosine; HC, high-dose (1\%) carnosine

${ }^{3)}$ Aspartate aminotransferase

${ }^{4)}$ Alanine aminotransferase

${ }^{\mathrm{a}-\mathrm{c}}$ Different superscripts in the same row indicate that the data are significantly different per Duncan's multiple range test $(p<0.05)$.

There was no significant difference in concentrations of total protein among the groups. Serum AST concentrations in the carnosine-supplemented groups were significantly higher than in the CON group $(p<0.05)$, and serum AST levels tended to increase as carnosine levels increased in the diet. There was no significant difference in serum ALT concentrations.

\section{Discussion}

In this study, carnosine showed the beneficial effects in vivo model. The supplementation of carnosine showed the positive dose-dependent relationship with muscle carnosine concentration of rats. This study also found that carnosine intake improved the lipid profile and blood biochemical indices of rats dose-dependently without significant change of body weight and food intake. No et al. (2004) also reported that oral administration of $25 \mathrm{mg} / \mathrm{kg}$ body weight of carnosine for eight weeks did not have an effect on body weight. These findings are consistent with several studies (No et al., 2004; Sato et al., 2008). Similarly, it is reported that beta-alanine supplementation increases the muscle carnosine and improves exercise capacity in elderly subjects (del Favero et al., 2012). Because carnosine and anserine are $55 \mathrm{mg} / 100 \mathrm{~mL}$ and $88 \mathrm{mg} / 100 \mathrm{~mL}$ boiled meat homogenate in chicken, respectively (Hermanuseen et al., 2012), carnosine could be used or developed as a functional food ingredient obtained from chicken meat.

Generally, carnosine levels are higher in fast-twitch (FT) muscles and lower in slow-twitch (ST) muscles (Bump et al., 1990; Dunnett et al., 1997). The gastrocnemius muscle usually contains a larger proportion of FT muscle fibers, while the soleus muscle primarily consists of ST muscle fibers. In the present study, mean carnosine levels in gastrocnemius muscles were higher than the mean carnosine levels in the soleus muscle of all groups In addition, rats fed the $\mathrm{HC}$ diet had the highest concentrations of carnosine in both the soleus and gastrocnemius muscles. Tamaki et al. (1984) reported that $0.9 \%$ dietary carnosine intake did not significantly increase carnosine levels in muscles. But, in the present study, $1 \%$ dietary carnosine intake significantly increased carnosine levels in both the soleus and gastrocenmius muscles. A study conducted by Maynard et al. (2001) found that only the highest carnosine dose (1.8\%), fed over a period of eight 
weeks, increased muscle carnosine content in the soleus muscle. Also, Tamaki et al. (1984) reported that 1.8\% dietary carnosine increased carnosine concentrations approximately twice as much in the gastrocnemius muscle when compared carnosine concentrations in the gastrocnemius muscles of the control group. Increased carnosine concentration in the muscles may result in improved athletic performance. $\beta$-Alanine, one component of carnosine, was provided to human male subjects for four and 10 weeks and resulted in increased carnosine concentrations in the vastus lateralis muscle and improved performance in a bicycle capacity test (Hill et al., 2007). These results are in line with findings by Suzuki et al. (2002) that showed positive correlations between carnosine concentrations and mean power per body weight during highintensity exercise (30S Wingate test).

In this study, the triglyceride level of the $\mathrm{HC}$ group was significantly lower than that of the CON group. Lee et al. (2005) reported that a dietary supplementation of 0.5 or 1 $\mathrm{g} / \mathrm{L}$ of carnosine in water caused a dose-dependent decrease in triglyceride concentrations in the liver and heart, and that $1 \mathrm{~g} / \mathrm{L}$ carnosine reduced total cholesterol levels in the liver and heart of streptozotocin-induced diabetic mice. However, in the present study, there was no significant difference in serum total cholesterol levels among the groups. Duthie and Bellizzi (1999) suggested that antioxidant supplementation might help to increase circulating HDL-cholesterol concentrations. Carnosine is thought to have antioxidant properties due to its ability to quench single molecular oxygen and scavenge superoxide radicals (Gariballa and Sinclair, 2000; Pavlov et al., 1993). Therefore, a carnosine supplement may have a beneficial effect on HDL-C concentrations in blood. In this study, all of the carnosine-supplemented groups had significantly higher concentrations of HDL-C than that of the CON group $(p<0.05)$, and the HDL-C levels tended increase with increasing amounts of supplemented carnosine.

All of the carnosine-supplemented groups in this study had significantly lower concentrations of LDL-C than that of the CON group $(p<0.05)$, suggesting that carnosine supplementation may decrease LDL-C in blood. Lipids are very susceptible to attack by free radicals and oxidized-LDL species (Takahashi et al., 2005). LDL molecules are the major cholesterol carriers in blood and appear to be involved in the development of atherosclerosis. Lee et al. (2005) found that carnosine effectively protected LDL against glucose-induced oxidation in humans by acting as a scavenger of free radicals in LDL and retarding LDL oxidation. It has also been suggested that carnosine $(3 \mu \mathrm{M})$ inhibits copper-promoted LDL $(20 \mu \mathrm{g}$ of protein $/ \mathrm{mL}$ ) oxidation (Decker et al., 2001), and effectively suppresses LDL oxidation by copper or peroxyl radicals in an ex vivo test (Bogardus and Boissonneault, 2000).

Wolford et al. (1986) indicated that the AST reference range for rats less than six months old was approximately 68-128 IU/L. Mean AST levels in this study were significantly higher than this reference range, with the exception of the CON group $(p<0.05)$. AST levels were highest in the HC group. ALT concentrations of all experimental groups tended to be higher than ALT concentrations in the CON group. Total bilirubin levels in the HC group were significantly higher than that of the CON group. These elevated bilirubin levels suggest that a dietary supplement of $1 \%$ carnosine over a period of four weeks may induce liver damage in rats. However, Liu et al. (2008) reported that ingestion of carnosine in water $(1 \mathrm{~g} / \mathrm{L})$ for four weeks did not affect AST levels. Several other studies have also shown that carnosine supplementation protects the liver against ethanol (Artun et al., 2010) and thioacetamide (Aydin et al., 2010), both of which are widely used to induce liver injuries in experiments. Carnosine also appears to protect the liver from acetaminophen (Yan et al., 2009), which can also cause liver injuries if administered above recommended dosages. That said, Ibrahim et al. (2008) indicated that moderate levels of dietary carnosine $(200 \mathrm{mg}$ or $1000 \mathrm{mg}$ carnosine/kg food) in rats had no significant effect on the incidence of myodegeneration and susceptibility of erythrocytes to hemolytic stress or oxidative stress in the liver. Sato et al. (2008) reported that the no-observed-adverse-effect level for rats fed chicken breast extract containing at least $35 \%$ carnosine and anserine was considered to be 2000 $\mathrm{mg} / \mathrm{kg}$ body weight/day. When the carnosine intake in this study is converted to that dose unit, the LC group consumed $8.7-12.1 \mathrm{mg} / \mathrm{kg}$ body weight/day of carnosine and the $\mathrm{HC}$ group was $870.8-1204.1 \mathrm{mg} / \mathrm{kg}$ body weight/ day of carnosine. Thus, results regarding the effects of carnosine supplementation on the liver of rats are still controversial.

In conclusion, the findings in this study suggest that high-dose (1\%) carnosine supplementation increases carnosine concentrations in muscles. Low levels of dietary carnosine supplementation ( $0.01 \%$ carnosine) may improve lipid profiles by lowering LDL-C and increasing HDL-C levels in blood. However, several biochemical indices used to indicate liver damage including AST, ALT, and total bilirubin increased with carnosine supplementation, 
particularly high-dose carnosine. Therefore, further research is needed to determine the effects of carnosine supplementation on the liver. Future studies should explore the minimum amount of carnosine required to increase carnosine concentrations in muscles and improve lipid profiles, without negatively impacting biochemical indices related to liver damage.

\section{Acknowledgement}

This research was supported by the Chung-Ang University Research Grant in 2012.

\section{References}

1. Aristoy, M. C., Soler, C., and Toldrá, F. (2004) A simple, fast and reliable methodology for the analysis of histidine dipeptides as markers of the presence of animal origin proteins in feeds for ruminants. Food Chem. 84, 485-491.

2. Artun, B., Küskü-Kiraz, Z., Gulluoğlu, M., Cevikbaş, U., Koçak-Toker, N., and Uysal, M. (2010) The effect of carnosine pretreatment on oxidative stress and hepatotoxicity in binge ethanol administered rats. Hum. Exp. Toxicol. 29, 659665.

3. Aydin, A. F., Küskü-Kiraz, Z., Doğru-Abbasoğlu, S., Güllüoğlu, M., Uysal, M., and Koçak-Toker, N. (2010) Effect of carnosine against thioacetamide-induced liver cirrhosis in rat. Peptides 31, 67-71.

4. Bailey, D. M., Davies, B., Young, I. S., Hullin, D. A., and Seddon, P. S. (2001) A potential role for free radical-mediated skeletal muscle soreness in the pathophysiology of acute mountain sickness. Aviat. Space Environ. Med. 72, 513-521.

5. Bogardus, S. L. and Boissonneault, G. A. (2000) Carnosine inhibits in vitro low-density lipoprotein oxidation. Nutr. Res. 20, 967-976.

6. Boldyrev, A. A. (1990) Retrospectives and perspectives on the biological activity of histidine-containing dipeptides. Int. J. Biochem. 22, 129-132.

7. Bump, K. D., Lawrence, L. M., Moser, L. R., Miller-Graber, P. A., and Kurcz, E. V. (1990) Effect of breed of horse on muscle carnosine concentration. Comp. Biochem. Physiol. A. Comp. Physiol. 96, 195-197.

8. Decker, E. A. (1995) The role of phenolics, conjugated linoleic acid, carnosine and pyrroloquinoline quinone as nonessential dietary antioxidants. Nutr. Rev. 53, 49-58.

9. Decker, E. A., Ivanov, V., Zhu, B. Z., and Frei, B. (2001) Inhibition of low-density lipoprotein oxidation by carnosine histidine. J. Agric. Food Chem. 49, 511-516.

10. del Favero, S., Roschel, H., Solis, M. Y., Hayashi, A. P., Artioli, G. G., Otaduy, M. C., Benatti, F. B., Harris, R. C., Wise, J. A., Leite, C. C., Pereira, R. M., de Sá-Pinto, A. L., LanchaJunior, A. H., and Gualano, B. (2012) Beta-alanine (Carnos$\mathrm{yn}^{\mathrm{TM}}$ ) supplementation in elderly subjects (60-80 years): effects on muscle carnosine content and physical capacity.
Amino Acids 43, 1-8.

11. Dunnett, M., Harris, R. C., Soliman, M. Z., and Suwar, A. A. S. (1997) Carnosine anserine and taurine contents in individual fibres from the middle gluteal muscle of the camel. Res. Vet. Sci. 62, 213-216.

12. Dunnett, M. and Harris, R. C. (1999) Influence of oral betaalanine and L-histidine supplementation on the carnosine content of the gluteus medius. Equine Vet. J. Suppl. 30, 499504.

13. Duthie, G. G. and Bellizzi, M. C. (1999) Effects of antioxidants on vascular health. Br. Med. Bull. 55, 568-577.

14. Friedewald, W. T., Levy, R. I., and Fredrickson, D. S. (1972) Estimation of the concentration of low-density lipoprotein cholesterol in plasma, without use of the preparative ultracentrifuge. Clin. Chem. 18, 499-502.

15. Gariballa, S. E. and Sinclair, A. J. (2000) Carnosine: physiological properties and therapeutic potential. Age Aging. 29, 207-210.

16. Guiotto, A., Calderan, A., Ruzza, P., and Borin, G. (2005) Carnosine and carnosine-related antioxidants: a review. Curr. Med. Chem. 12, 2293-2315.

17. Hermanussen, M., Gonder, U., Stegemann, D., Wesolowski, M., Ulewicz-Magulska, B., Wartensleben, H., and Hoffmann, G. F. (2012) How much chicken is food? Questioning the definition of food by analyzing amino acid composition of modern convenience products. Anthropol. Anz. 69, 57-69.

18. Hill, C. A., Harris, R. C., Kim, H. J., Harris, B. D., Sale, C., Boobis, L. H., Kim, C. K., and Wise, J. A. (2007) Influence of beta-alanine supplementation on skeletal muscle carnosine concentrations and high intensity cycling capacity. Amino Acids 32, 225-233.

19. Jackson, M. C. and Lenney, J. F. (1996) The distribution of carnosine and related dipeptides in rat and human tissues. Inflamm. Res. 45, 132-135.

20. Ji, L. L. (1995) Oxidative stress during exercise: implication of antioxidant nutrients. Free Radic. Biol. Med. 18, 10791086.

21. Klebanov, G. I., Teselkin, Y. O., Babenkova, I. V., Popov, I. N., Levin, G., Tyulina, O. V., Boldyrev, A. A., and Vladimirov, Y. A. (1997) Evidence for a direct interaction of superoxide anion radical with carnosine. Biochem. Mol. Biol. Int. 43, 99106.

22. Lee, Y. T., Hsu, C. C., Lin, M. H., Liu, K. S., and Yin, M. C. (2005) Histidine and carnosine delay diabetic deterioration in mice and protect human low density lipoprotein against oxidation and glycation. Eur. J. Pharmacol. 513, 145-150.

23. Liu, W. H., Liu, T. C., and Yin, M. C. (2008) Beneficial effects of histidine and carnosine on ethanol-induced chronic liver injury. Food Chem. Toxicol. 46, 1503-1509.

24. Mastaloudis, A., Leonard, S. W., and Traber, M. G. (2001) Oxidative stress in athletes during extreme endurance exercise. Free Radic. Biol. Med. 31, 911-922.

25. Maynard, L. M., Boissonneault, G. A., Chow, L. K., and Bruckner, G. G. (2001) High levels of dietary carnosine are associated with increased concentrations of carnosine and histidine in rat soleus muscle. J. Nutr. 131, 287-290. 
26. Nardone, A., Romanò, C., and Schieppati, M. (1989) Selective recruitment of high-threshold human motor units during voluntary isotonic lengthening of active muscles. J. Physiol. 409, 451-471.

27. No, J. C., Kim, H. T., Chae, C. H., Lee, S. H., and Ah, E. N. (2004) The effect of L-carnosine administration and aerobic exercise on blood lipid peroxidation and endurance performance ability in rats. Korean J. Exer. Nutr. 8, 79-85.

28. Petersen, E. W., Ostrowski, K., Ibfelt, T., Richelle, M., Offord, E., Halkjaer-Kristensen, J., and Pedersen, B. K. (2001) Effect of vitamin supplementation on cytokine response and on muscle damage after strenuous exercise. Am. J. Physiol. Cell Physiol. 280, C1570-C1575.

29. Pavlov, A. R., Revina, A. A., Dupin, A. M., Boldyrev, A. A., and Yaropolov, A. I. (1993) The mechanism of interaction of carnosine with superoxide radicals in water solutions. Biochim. Biophys. Acta. 1157, 304-312.

30. Plowman, J. E. and Close, E. A. (1988) An evaluation of a method to differentiate the species of orgin of meats on the basis of the contents of anserine, balenine and carnosine in skeletal muscle. J. Sci. Food Agric. 45, 69-78.

31. Sato, M., Karasawa, N., Shimizu, M., Morimatsu, F., and Yamada, R. (2008) Safety evaluation of chicken breast extract containing carnosine and anserine. Food Chem. Toxicol. 46, 480-489.

32. Suzuki, Y., Ito, O., Mukai, N., Takahashi, H., and Takamatsu,
K. (2002) High level of skeletal muscle carnosine contributes to the latter half of exercise performance during 30-s maximal cycle ergometer sprinting. Jpn. J. Physiol. 52, 199205.

33. Takahashi, Y., Zhu, H., and Yoshimoto, T. (2005) Essential roles of lipoxygenases in LDL oxidation and development of atherosclerosis. Antioxid. Redox Signal 7, 425-431.

34. Tamaki, N., Funatsuka, A., Fujimoto, S., and Hama, T. (1984) The utilization of carnosine in rats fed on a histidine-free diet and its effect on the levels of tissue histidine and carnosine. J. Nutr. Sci. Vitaminol. 30, 541-551.

35. Wolford, S. T., Schroer, R. A., Gohs, F. X., Gallo, P. P., Brodeck, M., Falk, H. B., and Ruhren, R. (1986) Reference range data base for serum chemistry and hematology values in laboratory animals. J. Toxicol. Environ. Health 18, 161188.

36. Yan, S. L., Wu, S. T., Yin, M. C., Chen, H. T., and Chen, H. C. (2009) Protective effects from carnosine and histidine on acetaminophen-induced liver injury. J. Food Sci. 74, H259$\mathrm{H} 265$.

37. Ibrahim, W., Tatumi, V., Yeh, C. C., Hong, C. B., and Chow, C. K. (2008) Effects of dietary carnosine and vitamin E on antioxidant and oxidative status of rats. Int. J. Vit. Nutr. Res. 78, 230-237.

(Received 2012.7.4/Revised 2012.8.31/Accepted 2012.9.6) 